events. It remains difficult to assess the frequency and importance of gene conversion in the maintenance and expression of antibody genes.

It is now clear that there are several hundred $V_{H}$ and $V_{L}$ gene segments, approximately $12 D$ segments, $5 J_{H}$ and $4 J_{x}$ segments in the mouse germ line. The relative contribution of this inherited diversity and the later expansion of the repertoire by combinatorial and junctional diversification varies for each system. Although the full extent of antibody diversity that can be obtained from a given set of $V$-, $D$-, $J$-gene segments remains obscure, data on antibody and gene structure reveal considerable flexibility in manner of their assembly, since multiple combinations and permutations of the germ-line genes are possible. Whether there are favoured com- binations and permutations or whether selection operates on an assortment of every possible configuration is still open to question. Furthermore, there is strong evidence that somatic mutation and/or gene conversion act on the rearranged $V$ genes.

But two unrelated questions remain. When does mutation occur in the differentiation of lymphocytes from committed precursors with rearranged genes to antibody-secreting cells? What is the relative contribution of mutation to the available antibody repertoires? At present it seems likely that high rates of mutation are associated with antigen-driven clonal expansion.

T.J. Kindt and $A$. Coutinho are at the Institut Pasteur, 28 rue du Dr Roux, 75724 Paris Cedex 15.

\title{
Embryology
}

\section{Lethal mutation in collagen gene}

\section{from Keith $R$. Willison}

RUDOLPH Jaenisch's group recently reported the first success in artificially inducing a mutation that halts mammalian embryogenesis at a specific stage of development ${ }^{1}$. Their technique, as discussed in News and Views at the time ${ }^{2}$, is to infect early mouse embryos with murine leukaemia retrovirus, the DNA of which becomes stably inserted into the genome of some of the animals. Mutant strains can then be bred from animals carrying such insertions and one of these, Mov-13, carries a recessive embryonic lethal: homozygous embryos die at day 13 of gestation. In Nature this week, Schnieke et $a l{ }^{3}$ report that they have identified the site of the mutation as the gene encoding $\alpha$ l(I) collagen. The exact mechanism of the lethal effect is not clear; but it may have interesting implications both for inherited disorders of collagen metabolism in man and for the future of retroviral mutagenesis as a tool for the exploration of mammalian development.

The discovery that the lethal mutation is in the gene encoding $\alpha 1$ (I) collagen was made possible by the availability of human and chicken cDNA clones that crosshybridize with mouse DNA ${ }^{4,5}$. The retroviral genome has integrated at the 5 ' end of the gene and blocks transcription. In normal embryos, abundant RNA transcripts from the $\alpha 1(\mathrm{I})$ collagen gene first become detectable at day 12 of gestation. In Mov-13 homozygous embryos, no stable $\alpha 1$ (I) transcripts are ever detectable and the embryos die from a general tissue necrosis that is first visible in the liver at day 12 of gestation.

The absence of malformations in the embryos suggests that type- 1 collagen plays no part in morphogenesis up to this stage (12-day mouse embryos have prominent limb buds just beginning to digitate from mesenchyme to precartilage for example). Since some type-1 collagen is serologically detectable in 9-day mouse embryos, Schneike et al. suggest that inhibition of type-1 collagen synthesis may interfere with the laying-down of the extracellular matrix which is thought to be important in interactions between mesenchyme and epithelium. The failure of cells to differentiate in the absence of such interactions may ultimately be responsible for the death of the whole embryo.

The Mov-13 mouse strain should be directly relevant to the study of human disease because collagen defects are strongly implicated in some heritable disorders such as osteogenesis imperfecta. This group of disorders is characterized by brittle bones but is otherwise very heterogeneous. A case that resulted in perinatal death of the patient was recently described in this journal. The patient was heterozygous for an internal deletion of 0.5 kilobases in the pro- $\alpha 1$ (I) collagen gene which resulted in an in-frame mRNA transcript coding for a truncated polypeptide ${ }^{6}$. The type-1 procollagen trimers containing truncated pro- $\alpha 1$ (I) chains are rapidly degraded and this results in a decrease in the number available for fibre formation. Since Mov$13 /+$ mice are healthy however, it seems to be possible to cope with half as much $\alpha 1$ (I) collagen so long as it is normal (assuming no compensatory synthesis from the wildtype allele). Thus insertion mutagens that can produce null alleles may in fact be the most effective agents for producing viable recessive mutants.

What does this result mean for mammalian developmental geneticists? It has recently been argued in Nature $e^{7}$ that mammalian developmental genetics cannot hope to compete with research on Drosophila whose morphogenetic mutants, to say nothing of its more manageable genome size, have lately been so elegantly exploited and for which a transformation system analogous to Jaenisch's retroviral mutagenesis has now been developed ${ }^{8}$.

In fact it is germane to the consideration of the collagen lethal mutation to discuss some estimates from Drosophila melanogaster of the number of genes required for any developmental process. Garcia-Bellido and Robbins ${ }^{9}$ have studied the viability of female germ-line cells homozygous for zygotic lethals in $D$. melanogaster. Using mitotic recombination they show that the germ line is more sensitive to mutations than clones of epidermal cells: more than twice as many of the zygotic lethals are lethal in the germ line than the epidermis. As 9 per cent of zygotic lethals are lethal in epidermal clones ${ }^{10}$ it is probable that more than 20 per cent of cell-autonomous genes are required for female germ-cell development. However, there are clearly many genes whose products are not and this class would include non-cellautonomous genes coding for extracellular matrix components.

Mov-13 was generated by infecting postimplantation embryos with virus and was the only insertion discovered in several hundred offspring, insertion being assayed by viraemia. Schnieke et al. speculate that the murine leukaemia virus provirus preferentially inserts into actively transcribed DNA, a testable hypothesis since it should be possible to show whether primordial germ cells synthesize a1(I) collagen. If this is the case, it reduces the target size since only 3 per cent of the genome is transcribed. Because collagen is a secreted product, the effects of mutations in the gene encoding it would be expected to be non-cell-autonomous. These considerations may thus explain why the collagen gene should have accepted an integrating provirus, and why it was possible to isolate the resulting mutant. It would have been all the more exciting to have discovered a new gene by Jaenisch's technique rather than a previously cloned, somatically transcribed one. However, we now know that a fairly early-acting recessive lethal mutation in mouse embryogenesis maps in the $\alpha 1$ (I) collagen gene and this is an important result for the field of developmental genetics.

Keith $R$. Willison is at the Chester Beatty Laboratories, Fulham Road, London SW3 $6 J B$.

\footnotetext{
1. Jaenisch, R. et at. Cell 32, 209 (1983).

Willison, K. Nature 300, 401 (1982).

3. Schnieke, A., Harbers, K. \& Jaenisch, R. Nature 304, 315 (1983).

4. Chu, M.L., Myers, J.C., Bernard, M.P., Ding, J.F. \& Ramirez, F. Nucleic Acids Res. 10, 5925 (1982)

5. Lehrach, H. et al. Biochemistry 18, 3146 (1979)

6. C hu, M.L. et al. Nature 304, 78 (1983).

7. North, G. Nature 303, 134 (1983).

Rubin, G.M. \& Spradling, A.C. Science 218, 348 (1982)

9. Garcia-Bellido, A. \& Robbins, L.G. Genetics 103, 235 (1983).

10. Ripoll, P. \& Garcia-Bellido, A. Genetics 91, 443 (1979)
} 